\title{
SIMULATION-BASED CAPACITY PLANNING AND SCHEDULING WITH AUTOSCHED
}

\author{
Michael B. Thompson \\ AutoSimulations \\ 655 Medical Drive \\ Bountiful, Utah 84011, U.S.A.
}

\section{ABSTRACT}

The function of 'Capacity Planning' is the process of determining in detail how much tooling, personnel, and equipment resources are required to accomplish specified product demand. The function of 'Scheduling' is the timesequenced allocation of resources such as machines, personnel, tools, and component materials to perform a collection of tasks to manufacture products.

Traditional methods of Capacity Planning and Scheduling have used infinite capacity and static time calculation approaches to these functions. The result of the past methods are inaccurate and non-representative answers to extremely important questions.

This paper introduces AutoSched as a tool for planning and scheduling personnel to help answer the questions of Capacity Planning and Scheduling.

\section{AUTOSCHED'S WORLD VIEW}

AutoSched sees the world as a number of stations. Stations can be machines, work benches, assembly positions, or any location where work is performed on a product. A group of stations that perform essentially interchangeable work is called a family. Every station belongs to a family, even if the family only contains a single station. Families share a common input queue and work list for parts waiting for service from one of the stations in the family. Each station can have one or more calendars associated with it. Calendars specify when stations are unavailable for work.

Lots flow between families in an AutoSched model. They consist of a quantity of pieces of a given type of part, and flow according to a routing that you define using manufacturing terminology. A routing consists of an arbitrary number of steps indicating the parameters for that lot, such as the station family, the setup, processing time required, and the operator class. These parameters define the operation performed on the part.

Lots can either start the simulation at the first step of their routing, or at their current step if they are already in process. As lots flow through the steps in the routing, they enter the family work list and queue for the family designated for that step. Idle available stations in the family wake up one at a time and execute the task selection rules associated with that particular station. Task selection rules are the criteria you use to determine which lot to work on next at any given station. The task selection rule either allows the station to pick a lot from the potential parts or to wait for a better choice. The status of operators, tools, components, and other constraints may be considered in a task selection rule.

As lots are simulated through their routing, AutoSched provides the option of generating a schedule file. If your interest is Capacity Planning, this file is not needed. If your interest is Scheduling, this file logs the simulated time at which all of the operations take place. This schedule can be viewed using the $\underline{A} u t o \underline{S} c h e d \underline{D}$ iagnostic Tool (asdt). In addition to the schedule, factory performance statistics are collected, indicating the efficiency expected if you operate your factory per AutoSched's schedule.

To use AutoSched, you must provide (3) basic types of input:

- Factory resources (Stations, Operators, Tools, Storages, Rules, Calendars)

- Products (Parts, BOM, Routings, Setup Matrix, Purchased Parts, Movement Itineraries)

- Demand (Factory Orders, Preventative Maintenance Work Orders)

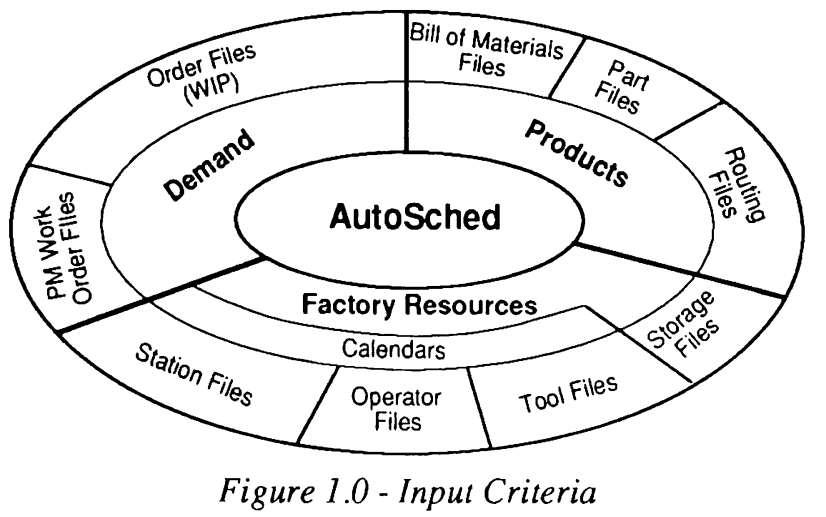


AutoSched is extremely flexible in that you only have to provide data that is available to you. For example, if you do not want to include the detail of modeling human operators, don't include it. In addition, AutoSched has defaults for almost all of the possible features.

\subsection{Data Requirements}

The data required for AutoSched can be entered from exiting data bases or spreadsheets, or it can be entered through AutoSched's powerful edit tables. An edit table allows the user to input or modify data in a stand-alone or integrated database fashion. The illustration below shows AutoSched's data definition user interface.

1. Data Organization - You simply organize the data to define the model; you don't program.

2. Decision Orientation - Stations, operators, and tools, rather than orders, make the decision of what to work on next. This reflects how decisions are made in the real world, i.e., by operators who can look at the whole system.

3. Calendar Capability - The simulation clock is converted to a calendar clock (Month, Day, Year, Hour, Minute, and Second). You can define an unlimited number of calendars, and attach them to equipment and personnel. Calendars include information such as scheduled maintenance and holidays.

4. Schedule Diagnostics - AutoSched provides Gantt charts and business graphs to help you visually interpret the schedules. Because AutoSched is based on AutoMod, AutoSimulations' 3-D graphical simulation software, animation also aids in the understanding of scheduling dynamics.

5. Customization - You can either use AutoSched's builtin scheduling rules or customize them using AutoMod's flexible simulation language.

6. Flexibility - With AutoMod and AutoSched, the same tool can be used for:

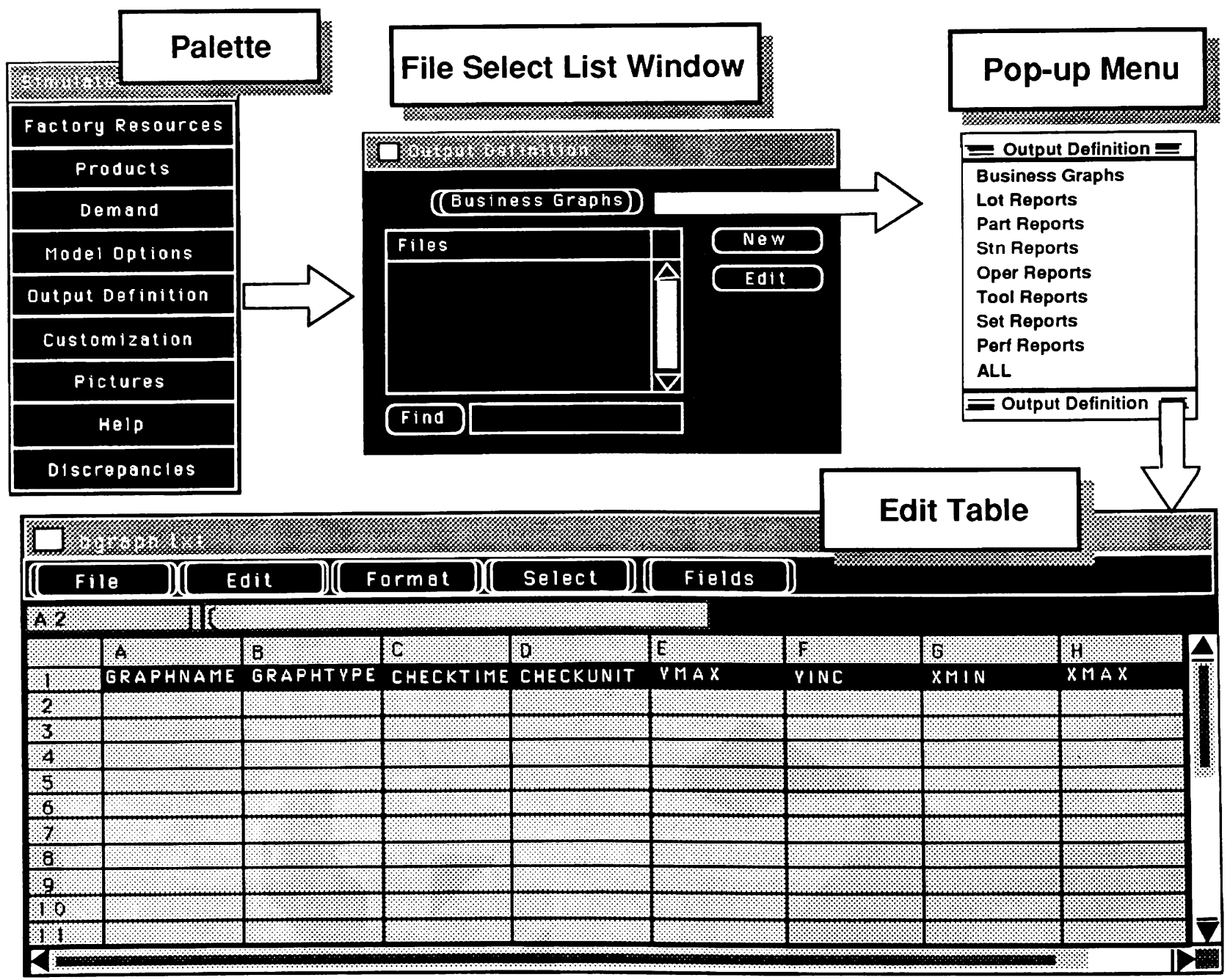

Figure 2.0 - AutoSched's Data Definiton User Interface 
- Factory Simulation

- Pre-planned schedule creation

- Real time dispatching

- Finite capacity planning and analysis

A scheduling or planning model requires data such as: Routing Definitions (for each part type), Station Definitions, Operator Definitions, Tool Definitions, Orders and WIP Status, and Calendar Definitions, among other data. Much of this information may exist in a database such as an MRP or a related CIM package. If it does exist, you don't need to duplicate the information, as it can be imported into AutoSched. The following is a brief explanation of AutoSched's files:

\section{Routing Definition}

The routing information describes the process steps of the different products. Each product type has its own routing or may share a routing.

\section{Station Definition}

Each station is described in this file. A station is defined as a place where work is performed. Stations are grouped into families. A family is a group of stations that share a common input queue.

\section{Operator Definition}

Each operator is defined in this file along with skill category and certification levels.

\section{Specific Tools Definition}

This information describes each of the factory's tools/fixtures.

\section{Order and WIP Status}

The order status file describes all orders scheduled during the simulation period and their current status. This information consists of both a current 'snapshot' of the shop floor and a list of the orders yet to be released.

\section{Calendar Definition}

Each resource (stations, operators, tools) can have its own working schedule. This work schedule can be composed of an unlimited number of calendars. This file describes the different types of calendars. Once they are defined, they can be attached to resources in the attachment file. There are five calendar types: shift, down, preventative maintenance (PM), holiday/exception, and reserved.

\section{Calendar Attachment}

A resource can have any number of calendars attached to it. The order in which calendars are attached to the resource dictates the order of precedence by which commonalities are resolved. For example, an operator might have a shift calendar, a holiday calendar, and a vacation calendar attached to it respectively. The holiday calendar overrides the common periods of the shift calendar, and the vacation calendar overrides the common periods with the other calendars. Calendar attachments allow a flexible, easy-to-maintain work schedule.

\subsection{Scheduling Rules}

AutoSched uses a revolutionary approach. This approach to scheduling rules makes it possible for plant scheduling personnel to define rules for each resource, such as stations and human operators. These rules are easy to construct, and are not limited to a single rule criterion or a single view of a resource.

A rule is a series of filters (Figure 3) into which potential tasks are fed. Each filter is a criterion, or test, the lots must pass. The filters screen out more and more lots until either one or zero lots remain for final selection.

You can utilize decision tree, sorting, and filtering capabilities in these rules. A rule can contain as many filters as needed.

This approach allows rules to consider multiple criteria in the decision process, thus allowing better decisions to be made.

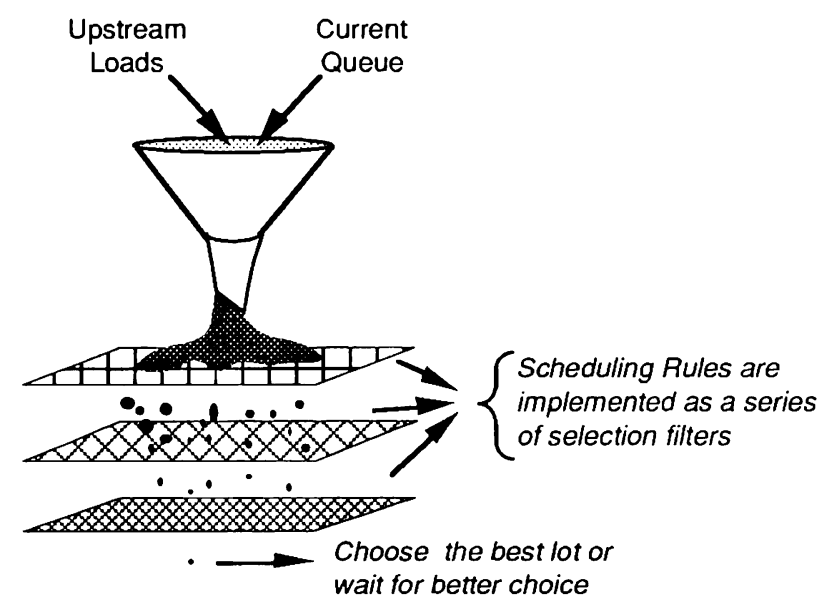

Figure 3 .0-Decision Fliter

\subsection{What does a Scheduling Rule look like and how does it work?}

Scheduling rules consist of one or more logic filters. Potential lots must pass through these logic filters to be selected. The following illustrations contain a scheduling task rule and a decision tree for a task rule. This example is simple. However, there is no limit to the level of sophistication that can be used in a scheduling task rule.

Example:

Suppose you must develop a scheduling task rule for a station where the amount of setup change-overs must be minimized for quality reasons or due to a lengthy setup time. When it's necessary to change to a new setup, the station should not select a lot or a batch of lots that requires a setup that is being used by any other station within the same family. If there is more than one lot or batch that meets the criteria, the one with the earliest due date is chosen. The decision tree demonstrates the filtering logic. 


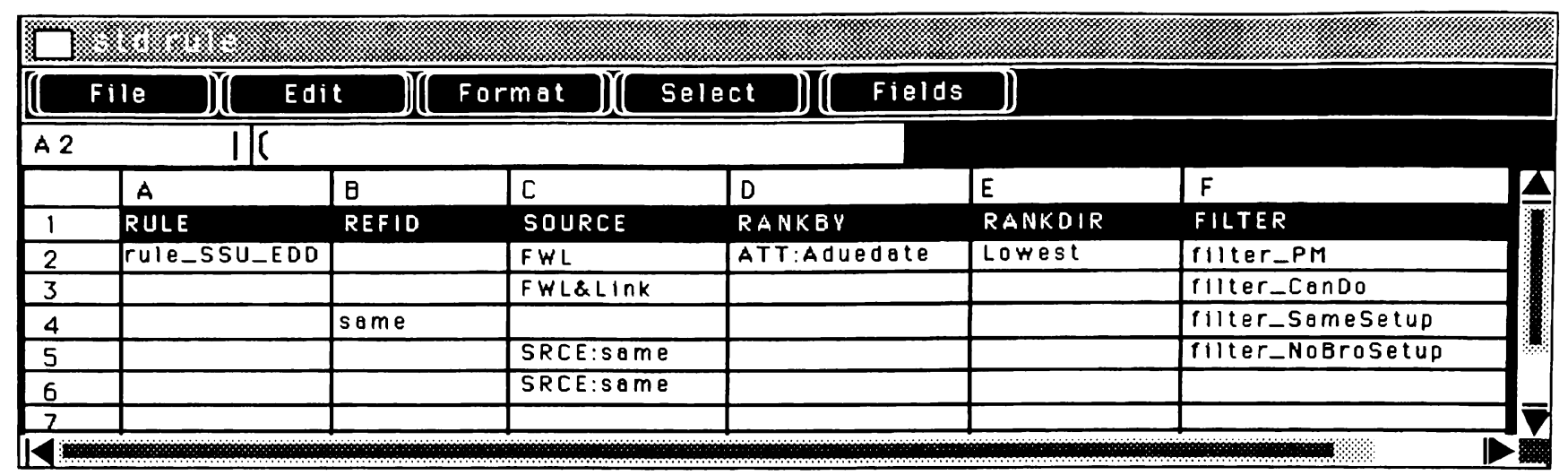

Figure 4.0 - Scheduling Task Rule

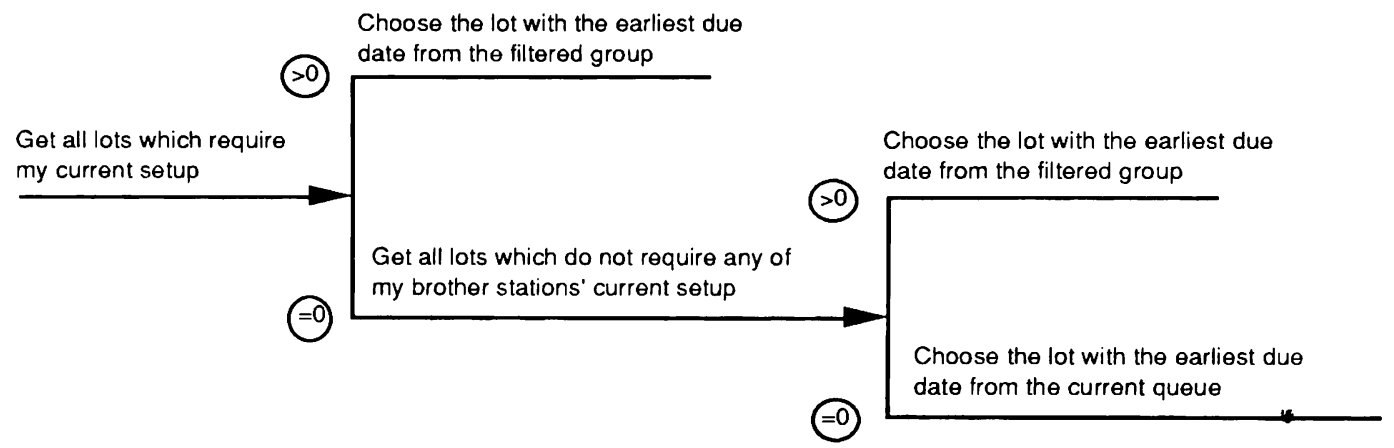

Figure 5.0 - Decision Tree

The station executes this logic when it finishes its previous work or after incoming work awakens it.

These rules do not guarantee the optimum schedule; no one can guarantee the optimum schedule due to the large number of possible combinations. However, through experimentation, rules can be developed that improve the performance of the factory on the order of $35 \%-60 \%$ (Norman, 1989).

Before developing scheduling rules, the factory's management must ask an important question: What is a good schedule?

\subsection{What is a Good Schedule?}

While this appears easy to answer, it's often more difficult to quantify than you might think. The attributes of a good schedule are:

- Minimum lead time

- On-time completion of all orders

- Minimum work-in-process and finished goods inventory

- Maximum resource utilization

- Minimum or no overtime

- Minimum cost in terms of the routing through alternate machines
While these are all worthy goals of a schedule, the fact is that some of them conflict. For example, assume your company just purchased a new, flexible machining center that cost $\$ 250,000$. To maximize the investment, the plant manager mandates that the new machining center must be utilized more than $90 \%$ of the available time.

To utilize equipment to that extent, there must constantly be a queue of work in front of $i t$, and when one order is completed another one must be waiting. Actually, several orders must be waiting, in case of unforeseen situations. In this example, the utilization goal conflicts with lead time, inventory, and possibly on-time completion goals, because products that must be serviced by the new machining center wait in a large queue.

Therefore, the answer to the question, "What is a good schedule?" is: conformance to management's goals and objectives. These goals and objectives must be developed by understanding inherent conflicts and the dynamics of relationships in a system. They can be measured by:

- Due date performance (the measure of earliness or lateness)

- Throughput (the number of products completed per unit time)

- Lead time (the amount of time orders stay in the system) 


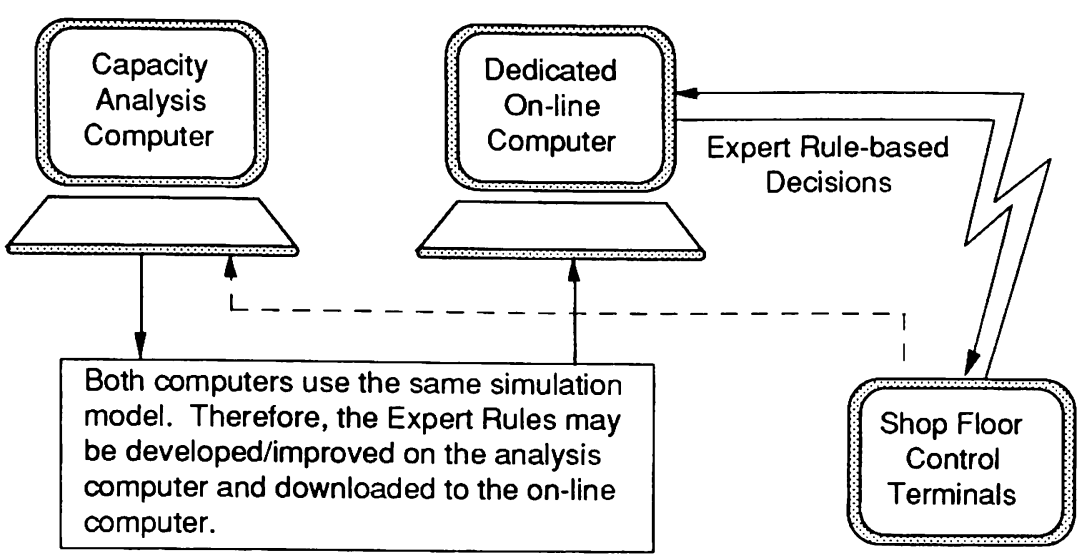

Figure 6.0 - Dispatching Flow Chart

tion. If unforeseen events occur in the real world, a new schedule must be prepared and provided to the shop floor. This mode, coupled with today's high performance scheduling software, allows planners to create 'work around' schedules with an ease and accuracy not previously available.

If the frequency of unforeseen events is high, the Pre-planned Scheduling mode may not be the best alternative. Unforeseen events can be a fundamental problem with pre-planned schedules.

- Real Time Dispatching is the second mode. It is a new extension to Simulationbased Scheduling.

Rather than create a new schedule each

- Inventory levels (WIP and Finished Goods)

- Resource utilization (the percent of time a resource is producing)

AutoSched easily computes these and other performance measures for each schedule produced.

\subsection{PRE-PLANNED SCHEDULING VS. REAL TIME DISPATCHING}

There are two ways to apply AutoSched and Coordinated Rule Based Scheduling to the manufacturing scheduling problem:

- Pre-planned Scheduling has been used most often. With Pre-planned Scheduling, a finite capacity schedule is developed from an accurate status of the shop floor and provided to the shop floor for implementatime an unplanned event occurs, the simulation is run parallel with real time. This method requires the model to receive detailed messages from the shop floor control system as events occur. These events are actual events rather than simulated events. The model updates the status of the internal data structures as it receives the event messages. When it receives an event message that necessitates task selection, the model performs the task selection and sends a message back to the shop floor system, telling it the next job to run on the selecting machine.

Machine failure events are received in the same manner as regular operation start and completion events. Even in dynamic environments, the model reacts accordingly and schedules the factory efficiently. With the capability of Real Time Dispatching, there is still

\section{Gantt Chart by Order}

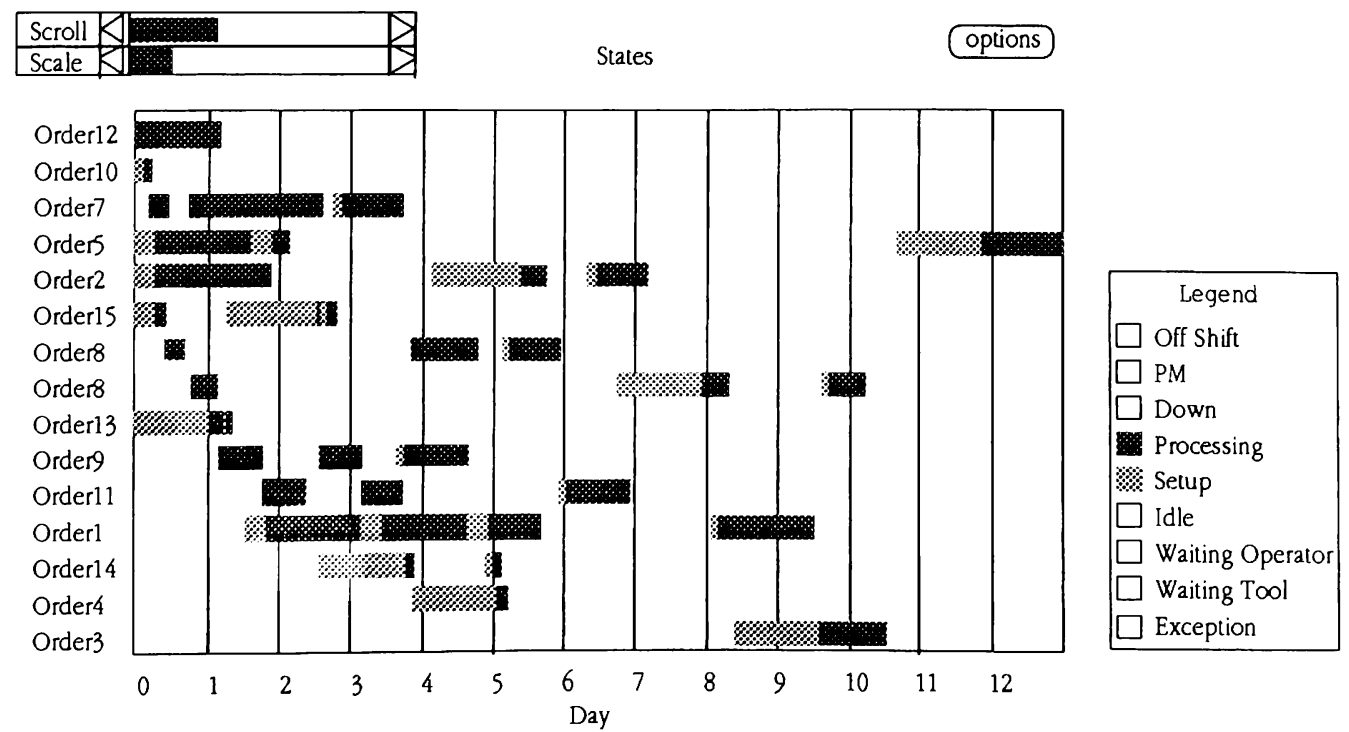

Figure 7.0 - Sample Gantt Chart 
the need for pre-planned scheduling and traditional capacity analysis. Therefore, one method does not preclude the other.

\subsection{AUTOSCHED OUTPUT}

There are two types of output from the simulation/ scheduling run: graphical and statistical.

- Graphical Output - You can define business graphs to track any statistic with a timeline graph, bar chart, or pie chart. These graphs/charts are updated as the model progresses. AutoSched also creates an interactive Gantt chart that allows you to see the simulation history for each resource and order. You can use the mouse to select an event on the Gantt chart and get detailed information about that event, such as the quantity of orders in that station's queue when the order was selected, the quantity of orders in the next step's queue, etc.

- Statistical/history reports - These include the Master Schedule file, Performance Report, Station Report, and any other user-defined reports.

The Master Schedule file contains either all, "step begin," "setup change," or "step complete" events. This schedule can then be post-processed to include only the desired information. For example, if you want the schedule for one area, you can pull out the information about that one area. Figure 8 is an example of a dispatch list created from the Master Schedule File.

Factory Performance Measures such as queue statistics, station utilization percentages, current WIP inventory levels, on time delivery performance, etc., are updated during the simulation, and can be displayed graphically or in printed form during and after the run. They are also summarized in a final report. For example, the Station Report details the percent of time each station spent in different states. You can modify what goes into the final reports.

\subsection{STEPS IN IMPLEMENTING SIMULATION- BASED SCHEDULING}

\subsection{Functional Requirements Definition and Scheduling Strategy}

Prior to beginning the implementation of a simulation model, it is important to establish the goals and objectives of the system. Tangible measures of schedule performance must be defined. These measures should be the standards used to manage the factory today.

Be aware that many measures of schedule performance are conflicting in nature. For example, a goal to minimize Work-In-Progress (WIP) and another goal to maximize equipment utilization are conflictive. A manufacturing facility is somewhat like a balloon. If you squeeze one section of the balloon, other sections bulge. If you maximize a single goal in a factory, you can easily accom-

\begin{tabular}{|c|c|c|c|c|c|c|c|}
\hline \multirow{2}{*}{\multicolumn{8}{|c|}{$\begin{array}{l}\text { Station: LMSO } \\
\text { Schedule Period }\end{array}$}} \\
\hline & & & & & & & \\
\hline \multicolumn{8}{|c|}{$\begin{array}{l}\text { Schedule Period } \\
\text { Date: }\end{array}$} \\
\hline \multicolumn{8}{|c|}{ Shift: 1st $(8: 00-16: 30)$} \\
\hline \multirow{3}{*}{$\begin{array}{l}\text { Order \# } \\
\text { H0069 }\end{array}$} & Lot\# & QTY & Part \# & Tools Req'd & Work Type & Start Time & End Time \\
\hline & 01 & 4000 & 2096421-1ML & $2096421-1$ & Processing & last shift & 09:34 \\
\hline & & & & OF 2096421-1 & & & \\
\hline \multirow[t]{3}{*}{ H9457 } & 01 & 50 & 2035432-1ML & $2035432-1$ & Setup-4inch & $09: 34$ & $10: 34$ \\
\hline & & & & OF 2035432-1 & Processing & $10: 34$ & $13: 56$ \\
\hline & & & 2CF 2035432-1 & & & & \\
\hline \multirow[t]{2}{*}{ L7546 } & 02 & 115 & $0877521-2$ & CF $0877521-2$ & Setup-6inch & $13: 56$ & $15: 56$ \\
\hline & & & & & Processing & $15: 56$ & $20: 14$ next shif \\
\hline
\end{tabular}

Figure 8 - Sample Dispatch List

\begin{tabular}{|c|c|c|c|c|c|c|c|c|c|c|c|c|c|c|c|}
\hline \multicolumn{6}{|c|}{ Week Ending: 04/11/92 } & \multicolumn{7}{|c|}{ Queue Statistics } & \multicolumn{3}{|c|}{$\begin{array}{l}\text { Production } \\
\text { Oty }\end{array}$} \\
\hline $\begin{array}{l}\text { Station BROC } \\
\text { Pleces }\end{array}$ & SETI & 1 OEF & $\mathbf{P M}$ & DWN & OPER & IDLE & Curr & Max & Avg & Axgl & brs) & Cur & $\operatorname{Max}$ & Avg & Lots \\
\hline LMS097 12 & 5 & 71 & 0 & 0 & 0 & 12 & 1 & 2 & 1.17 & 0.19 & 115 & 252 & 147.4 & 3 & 378 \\
\hline LMA1159 & 3 & 71 & 0 & 0 & 0 & 17 & 0 & 1 & 0.18 & 0.19 & 0 & 50 & 9.5 & 1 & 50 \\
\hline LMS987 24 & 1 & 50 & 0 & 0 & 0 & 25 & 3 & 6 & 2.52 & 2.13 & 756 & 1591 & 668.2 & 3 & 795 \\
\hline
\end{tabular}


plish it at the expense of other goals. Goals must be coordinated so that the best overall compromise is achieved.

Some typical measures of schedule performance are:

- Due date performance (the measure of earliness or lateness )

- Throughput (the number of products completed per unit of time)

- Lead time ( the amount of time orders or lots stay in the system)

- Inventory levels (Raw Material, WIP, and Finished goods)

- Resource utilization (the percent of time a resource is producing)

The challenge is to maximize these goals as a whole, not individually. The solution is to improve your factory's performance by implementing scheduling rules that yield the best composite schedule performance possible.

\subsection{Base Model Development}

The model should be developed and tested off-line with a representative set of data that is indicative of the real system.

\subsection{Rule Customization}

Rules should be developed off-line with a representative set of data that is indicative of the real scheduling problem. Usually it is not effective for each resource to try to optimize its own utilization. It is better to coordinate all of the stations and operators in the factory to work in concert. This is called Coordinate RuleBased Scheduling. The general strategy of Coordinated Rule-Based Scheduling follows:

First-Identify the critical resources in the factory. The critical resources are the ones that increase the overall throughput of the facility when their constraints are relaxed. Analyze production loading to determine the resources with the greatest load. Simulation does a great job of providing statistics on resources that have the highest utilization, and the largest number of lots waiting in queue, and the longest average wait time in queue.

Second- Keep critical resources busy. Do this by keeping manageable levels of work in the resources input queue, choosing tasks that minimize setups, and avoiding unnecessary setups. If necessary, critical resources should look upstream in an attempt to wait a reasonable amount of time for a better choice, rather than selecting from what is available on the family worklists. In some cases, resources should look down stream in an attempt to keep products flowing by choosing jobs that won't get bogged down in a large downstream queue.

Third- Identify server resources. Server resources have low to moderate work loads and can be used to aid critical resources. Server resources can insure that critical resources have work that minimizes setup time. Server resources should look downstream and feed the most productive work to critical resources.

Fourth - Run the model and analyze the results. Analyze the schedule performance measures established in your scheduling strategy. Pay particular attention to the utilization of critical resources. Were they full utilized? Were they spending an inordinate amount of time setting up? Also, check to see whether new critical resources have surfaced. If you see a trend that a rule change could help, enhance the rule and run the model again.

Fifth - Repeat the previous steps until you are satisfied with the schedule performance.

Sixth - Employ the selected rules with live data to schedule the facility. There may be some additional modifications to rules due to unforeseen issues that were not encountered in the test data.

\subsection{Output Customization}

Companies have metrics that are used as measures of performance to manage and gauge decisions in the facility. These same metrics should be reflected in the model output reports. AutoSched provides a powerful report-writing capability.

\subsection{Data Extraction and Integration}

Data extraction and integration is one of the most important aspects of a successful installation. AutoSched's flexible input data design facilitates the extraction effort from existing data systems. Generally, either users or AutoSimulations writes data extraction application programs that run automatically to load the AutoSched model with WIP status, equipment status, routings, and demand data. This is generally a very straightforward task.

\subsection{Model Validation and Verification}

Validating the model's behavior against real world factory performance is a critical step in having the users gain confidence in the model's ability to provide realistic information. The model should be run in parallel to the real world system using the same task selection criteria that is used. The real world system performance and the model's output should be compared and analyzed. Model adjustments may be required to reflect reality. Often, it is learned that the input data is wrong and new time standards are required.

\subsection{Capacity Planning Usage}

Capacity planning is often the first on-line usage of the technology. After the users gain confidence in the model, on-line scheduling uses should follow. 
4.8 Short Interval Scheduling (Pre-Planned)

As was described in section 2.0

\subsection{Real Time Dispatching}

As was described in section 2.0

\section{SUMMARY}

AutoSched with Coordinated Rule Based Scheduling is producing results. A major international semiconductor manufacturer recently reported that as a result of this technology, they reduced average cycle time from 25 days to 5 days. They also cut average work-in-process inventory to one fifth.

"It wasn't just the software that allowed us to make these improvements. It was the confidence that the software provided management that allowed them to cut inventories to previously unheard of levels," says a spokesperson for the semiconductor manufacturer.

Today manufacturers are looking for ways to be more responsive to customer needs, improve the time to market, and maximize utilization of resources that are currently available. AutoSched provides a means of increasing the productivity of factories without adding personnel or equipment.

\section{REFERENCES}

Thompson, M.B., 1990, Æ "Simulation Based Scheduling" Technical Report \# MS90 - 348. Society of Manufacturing Engineers, presented at the International Manufacturing Technology Conference (Chicago, Illinois, Sept. 7).

Rickel, J., 1988 "Issues in the Design of Scheduling Systems," Expert Systems and Intelligent Manufacturing - Michael D. Oliff Editor. Elsevier Science Publishing Co., Inc., New York, New York.

Conway, R. W., Maxwell,1967 W. L., and Miller, L. W., Theory of Scheduling. Addison-Wesley, Reading, Mass.

Norman, T. A., 1989 "Tailoring Dispatching Rules for Job Shop Scheduling," A White Paper Developed in the Department of Computer Science at Brigham Young University, Provo, Utah.

Thompson, M.B., 1992, "AutoSched Finited Capacity Scheduling and Planning" A White Paper.

Nichols, J. C., Thompson, M.B., 1993, "The Application of Finited Capactiy Planning and Scheduling toj Semiconductor Testing and Finishing Organizations," first presented at Semocon West '93 sponsered by SEMI (Semiconductor equipment and Materials International)

\section{BIOGRAPHY}

Mr. Thompson is a member of the society of Manufacturing Engineers, American Production and Inventory Control Society (CPIM), and The Society for Computer Simulation. He has authored papers on Finite Capacity Scheduling, Simulation-based Scheduling, The marriage of Simulation and Real Time Control, Generative Process Planning, SQC Methods for Machine Tool Acceptance, and Group Technology. He is a corporate Vice President and is responsible for scheduling products at AutoSimulations. He was previously employed by Texas Instruments, where he was the manager of Methods and Tooling, Manufacturing Manager, and an Automation Systems Engineer. He has been involved with modeling manufacturing systems for over 13 years. He holds B.S. and M.S. Degrees from the Department of Engineering Sciences and Technology at Brigham Young University. His research interests are in manufacturing and production systems, with emphasis in applied scheduling. 